\title{
Leveraging Implicit Contribution Amounts to Facilitate Microfinancing Requests
}

\author{
Suhas Ranganath ${ }^{*}$ \\ Walmart Labs \\ suhas.ranganath@walmartlabs.com
}

\author{
Ghazaleh Beigi \\ Arizona State University \\ gbeigi@asu.edu
}

\author{
Huan Liu \\ Arizona State University \\ huan.liu@asu.edu
}

\begin{abstract}
The emergence of online microfinancing platforms provides new opportunities for people to seek financial assistance from a large number of potential contributors. However, these platforms deal with a huge number of requests, making it hard for the requesters to get assistance for their financial needs. Designing algorithms to identify potential contributors for a given request will assist in satisfying financial needs of requesters and improve the effectiveness of microfinancing platforms. Existing work correlates requests with contributor interests and profiles to design feature based approaches for recommending projects to prospective contributors. However, contributing money to financial requests has a cost on contributors which can affect his inclination to contribute in the future . Literature in economic behavior has investigated the manner in which memory of past contribution amounts affects user inclination to contribute to a given request. To systematically investigate whether these characteristics of economic behavior would help to facilitate requests in online microfinancing platforms, we present a novel framework to identify contributors for a given request from their past financial information. Individual contribution amounts are not publicly available, so we draw from financial modeling literature to model the implicit contribution amounts made to past requests. We evaluate the framework on two microfinancing platforms to demonstrate its effectiveness in identifying contributors.
\end{abstract}

\section{CCS CONCEPTS}

- Information systems $\rightarrow$ Collaborative and social computing systems and tools; Reputation system; • Social and professional topics $\rightarrow$ Information system economics;

\section{KEYWORDS}

Crowdfunding, Socioeconmics, Q\&A, Information Seeking

ACM Reference Format:

Suhas Ranganath, Ghazaleh Beigi, and Huan Liu. 2018. Leveraging Implicit Contribution Amounts to Facilitate Microfinancing Requests. In Proceedings of WSDM 2018: The Eleventh ACM International Conference on Web Search

*The paper was submitted when the author was a graduate student at Arizona State University.

Permission to make digital or hard copies of all or part of this work for personal or classroom use is granted without fee provided that copies are not made or distributed for profit or commercial advantage and that copies bear this notice and the full citation on the first page. Copyrights for components of this work owned by others than ACM must be honored. Abstracting with credit is permitted. To copy otherwise, or republish, to post on servers or to redistribute to lists, requires prior specific permission and/or a fee. Request permissions from permissions@acm.org.

WSDM 2018, February 5-9, 2018, Marina Del Rey, CA, USA

(c) 2018 Association for Computing Machinery.

ACM ISBN 978-1-4503-5581-0/18/02 . \$ \$15.00

https://doi.org/10.1145/3159652.3159679

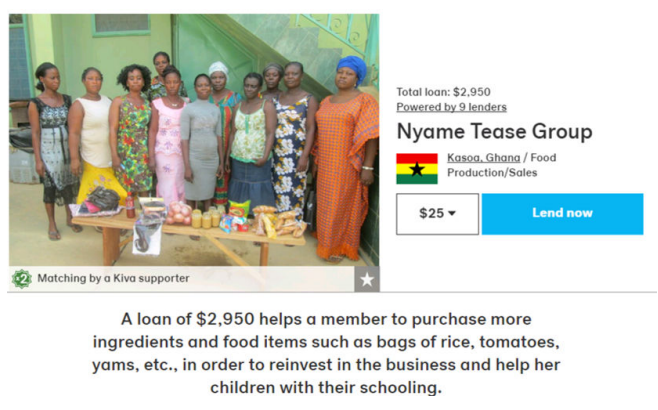

Figure 1: Request made on the microfinance platform Kiva

and Data Mining (WSDM 2018). ACM, New York, NY, USA, Article 4, 9 pages. https://doi.org/10.1145/3159652.3159679

\section{INTRODUCTION}

The rise of online financial platforms is being utilized by people to request, borrow and contribute to loans to fulfill their various financing needs. Microfinancing sites have emerged as a popular source for people to secure funding for a business venture or personal needs. Considerable interest has been shown in recent literature $[1,6,12]$ regarding the use of online microfinance platforms by primarily low-income people to seek to fund for their various financial and entrepreneurial needs [4].

Fig 1 illustrates an example of a request posted in the microfinance platform Kiva seeking help for their financial needs. In the example, a request is made by a non-governmental organization in Ghana called the Nyame Tease Group. It is seeking money to finance the purchase of food and education for the children of one of their members. The funding, in turn, would enable her to use existing funds for her business. The figure also features the set of contributors who contributed to the request. This example showcases the use of microfinancing platforms by low-income individuals for requesting loans to address their financial needs.

However, microfinancing platforms receive a high frequency of requests, and this makes it difficult for requesters to get their financial needs addressed [3]. Potential contributors have many requests on their wall, and they might not notice requests. The presence of a few highly visible requests [30] can inhibit others posted during the same time from getting noticed. Automatically identifying responders for financial requests can help the people to fulfill their financial needs and also enhance the effectiveness of micro-financing platforms.

The problem of identifying suitable contributors to financial requests faces several challenges. First, fulfilling financial requests has a cost on contributors which can alter his inclination to respond 
to future requests. The cost of response less prominent in informational requests, hence criteria for identifying information providers like relevance, expertise may not be sufficient here. Second, the amount contributors contributed to past requests in microfinancing platforms are not publicly available, making it hard for researchers to assess future contributing decisions based on them. Third, online microfinance platforms have a high number of requests in a given time each having many candidate contributors, leading to significant issues of scalability.

Economic research on the behavior of contributors to charity [10] emphasizes the importance of previous costs to contributors, as measured by the amounts they contributed in determining their future participation. The effect of past amounts is also explored in research on consumer behavior [26] where past prices consumers pay for commodities has been shown to shape their inclinations on future purchases. Drawing concepts from the literature on the economic behavior, we propose to leverage the effect of past contribution amounts on his inclination to contribute in the future to identify suitable responders to a given micro-financing request. Specifically, we answer the following questions: How to estimate the inclination of users to contribute to a given request from the amounts they contributed in the past? How to design financial models from the implicit past contribution amounts to identify potential contributors? The major contributions of the work are

- Formally defining the problem of identifying potential contributors to a request in online microfinance platforms;

- Proposing a framework to leverage implicit past contribution amounts to identify responders to financial requests; and

- Presenting extensive experimental evaluations of the framework on real-world datasets of requests posted on two microfinance platforms.

In this paper, we propose a framework that leverages financial information from the past contribution amounts to identify potential contributors for a given request. Drawing concepts from financial modeling literature, we model the effect of past contribution amounts of the user to estimate his inclination to contribute to a given request. We then utilize the financial models to identify contributors for a given funding request in online microfinance platforms.

\section{PROBLEM STATEMENT}

In this section, we present the notations and formally state the problem of facilitating requests in online microfinancing platforms We first present the set of notations used in the paper. Matrices are denoted by boldface uppercase letters (e.g. X), vectors by lowercase letters (e.g. $\mathbf{x}$ ) and sets can be denoted by $\mathcal{X}$. $\mathrm{X}_{i j}$ signifies the element in the $i^{t h}$ row and $j^{t h}$ column of matrix $\mathbf{X}$.

The proposed framework with the input, the information we employ and the expected output is illustrated in Figure 2. Let the request for funding be denoted by $r$. Each request is accompanied by a description outlining the reasons the funding is being requested. We denote the description by request word vector $q \in \mathbb{R}^{1 \times w}$ where $w$ is the total number of words. Each element of the vector is set to 1 if the word is present in the description and 0 if not. Let the overall set of potential contributors be denoted by $C$.
Each contributor in $C$ posts a description outlining his interests. We denote the description vector of each contributor as $\mathbf{c} \in \mathbb{R}^{1 \times w}$, where $w$ are the total number of words. In addition to this, each contributor might have funded previous requests in the past, and this can be used to provide insight into his preferences. For each contributor, we denote the word vector of the previous requests he has answered by $\mathbf{p} \in \mathbb{R}^{1 \times w}$, where $w$ is the total number of words. Let us denote information from the past requests which were fulfilled as $\mathcal{P}$. The includes the requested amount vector $\mathbf{r} \in \mathbb{R}^{q \times 1}$ where $q$ is the number of past requests, and the past request matrix $\mathrm{R} \in \mathbb{R}^{q \times C}$, where $q$ is the number of requests and $C$ is the number of contributors, where $\mathbf{R}_{i j}=1$ if contributor $j$ contributed to request $i$ and 0 otherwise.

Having described the notations, we now formally state the problem as follows "Given a request for funding $r$, the request word vector $\mathrm{q}$, the set of candidate contributors $C$, the contributor word vector $\mathbf{c}$ of each candidate contributor, the previous requests word vector $\mathbf{p}$ of each candidate contributor, information from the past requests $\mathcal{P}$, find the list of contributors for the given request."

In this section, we defined the notations and came up with a detailed problem statement. We next provide a detailed description of the framework designed to address this problem.

\section{THE PROPOSED FRAMEWORK}

Here, we first present measures for interests of the candidate contributors to the given request using their description and the requests they responded to in the past. We draw from financial modeling literature to leverage the past implicit contribution amounts to identify contributors to a microfinance request. We finally present the complexity analysis of the algorithm to evaluate its scalability in large-scale data prevalent in online microfinancing platforms.

\subsection{Measuring Contributor Interests}

Research on identifying responders to online requests have proposed several measures to estimate the correlation of the candidate respondents with the request[21,29]. We draw from these research to present metrics to estimate the contributor correlation using his interests and past response behavior. Let the word vector of the request be known as $\mathbf{q} \in \mathbb{R}^{1 \times w}$ and the description word vector of the candidate contributor be given by $\mathbf{c} \in \mathbb{R}^{1 \times w}$ where $w$ is the number of words. To represent the relationship between the given request and the description of the contributor, we transform the corresponding word vectors into a common latent dimension space using $\mathrm{S} \in \mathbb{R}^{\mathrm{n} \times \mathrm{w}}$. Here $n$ is the number of dimensions of the space $(\mathrm{n} \ll \mathrm{w})$. We can then represent the given request $r$ and contributor interest $c$ in the low dimensional space by $\mathrm{qS}^{T}$ and $\mathrm{CS}^{T}$ respectively. We represent the interest of the contributor using his relationship between the request and his description by incorporating dimension correlation with $T \in \mathbb{R}^{\mathrm{n} \times \mathrm{n}}$ as

$$
f=\mathrm{qS}^{\mathrm{T}} \mathrm{TSc}^{\mathrm{T}} .
$$

The interests of the contributor can also be determined by the previous requests towards which he contributed. Let $\mathbf{p} \in \mathbb{R}^{1 \times W}$ determine the previous requests answered by contributor $c \in C$, where $w$ is the total number of words. We follow a similar procedure to model the correlation of the contributor with the given request 

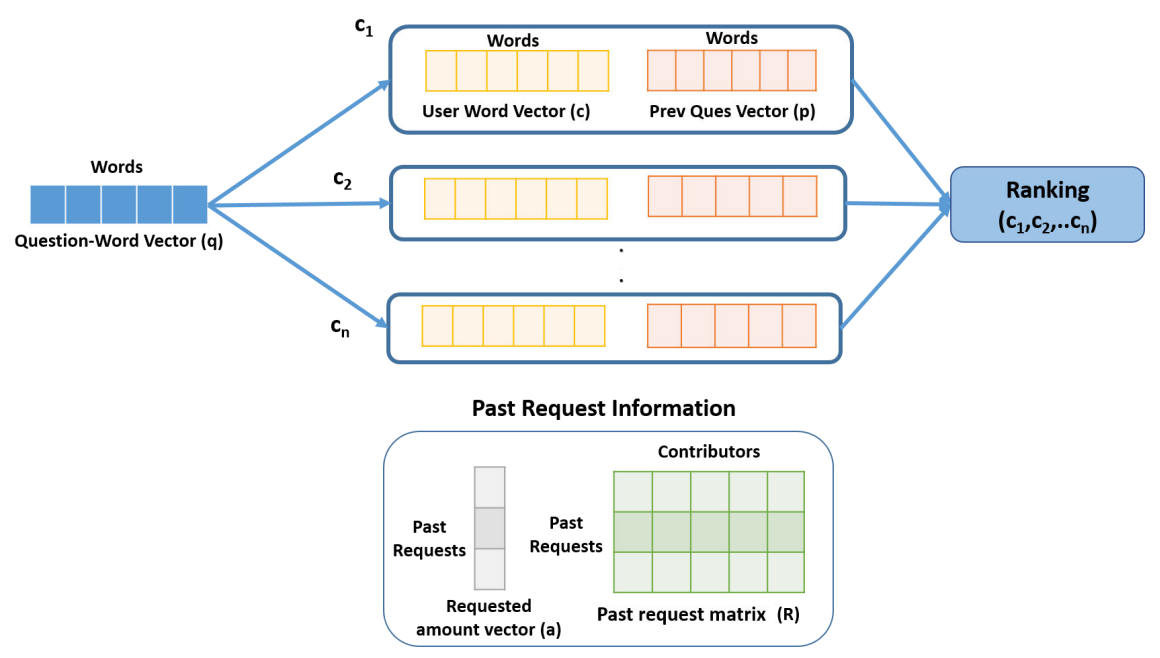

Figure 2: The proposed framework to facilitate funding requests in online microfinancing platforms

from previous requests he contributed to as

$$
f_{2}=\mathrm{qS}^{\mathrm{T}} \mathrm{TSp}^{\mathrm{T}} \text {. }
$$

We compute the overall interests of the contributor with the given request as $f=\mathbf{q S}^{\mathrm{T}} \mathbf{T S k}^{\mathrm{T}}$, where $\mathbf{k}=\mathbf{c}+\mathbf{p}$.

\subsection{Leveraging Implicit Contribution Amounts}

Contributing to financial requests bears a cost to the user, and hence the inclination of the user to contribute to a given financial request is effected by his past contribution amounts. We now design financial models to estimate the user inclination from past contribution amounts and user interest measures. User inclination to contribute to a given request can be affected by a host of factors not observable in the platform [25], hence we model the variation of user inclination over time as a stochastic process.

Let the joining time of a contributor $i$ be $t_{0}$ and the initial value he can contribute be given by $s_{0}$. We employ a stochastic variation process for modeling the user inclination. The following differential equation gives the stochastic variation of the user inclination of contributor $i$ from the past contribution amount $s_{i}$ and correlation with the request as

$$
\frac{d s_{i}}{s_{i}}=\mu_{i}\left(1+\mathrm{qS}^{\mathrm{T}} \mathbf{T S k}^{\mathrm{T}}\right) d t+\sigma_{i} d W,
$$

where $f_{i}=\mathrm{qS}^{\mathrm{T}} \mathrm{TSk}^{\mathrm{T}}$ computes the interests of the contributor to the request, $\mu_{i}$ is the drift which governs the deterministic component of his inclination to contribute, $\sigma_{i}$ is the variation, and $W$ is the Wiener process that accounts for the stochastic variation[23]. The deterministic component in the loan $u_{i}$ weighted by his interest in the loan. The deterministic and stochastic component $u_{i}$ and $\sigma_{i}$ is updated for each previous transaction of the user, and models how his inclination is affected by the transaction amounts .

a Solving this differential equation, the contribution amount of contributor $i$ for a past request that was posted at time $t$ is

$$
s_{i}=s_{0} \exp \left(\left(\mu_{i} \mathbf{n}_{i}-\frac{\sigma_{i}^{2}}{2}\right) t+\sigma_{i} W\right),
$$

From the properties of the Geometric Brownian Motion [18], we get $\ln (s) \sim \mathcal{N}(m, v)$. Here $\mathcal{N}$ denotes a normal distribution whose mean and variation is given by

$$
m_{i}=\ln \left(s_{0}\right)+\left(\mu_{i} \mathbf{n}_{i}-\frac{\sigma_{i}^{2}}{2}\right) t_{i}, v_{i}=\sigma_{i}^{2} t
$$

$\mathbf{n}_{i}=1+\mathrm{qS}^{\mathrm{T}} \mathrm{TSk}^{\mathrm{T}}$ The estimated mean contribution amount increases with greater interest in the question and is offset by the parameters learned from his previous transactions.

In online microfinance platforms the ground truth for the individual contribution amounts of past requests are not publicly known, but the total requested amount is known. We compute the optimal values of the parameter set $\mathcal{K}=\left\{\mu_{i}, \sigma_{i}, \mathrm{~S}, \mathrm{~T}\right\}$ by maximizing the log-likelihood between the real and the total implicit contribution amounts. To obtain the estimated requested amount, we follow a similar procedure for all the contributors of the past request. Combining the amounts of by all the contributors, and assuming independence, we get the estimated requested amount drawing from the normal random variable $\mathcal{N}(m, v)$, whose mean and variance is given by

$$
m=\sum_{i \in C} \ln \left(s_{0}\right)+\left(\mu_{i} \mathbf{n}_{i}-\frac{\sigma_{i}^{2}}{2}\right) t_{i}, v=\sum_{i \in C} \sigma_{i}^{2} t
$$

We then equate the estimated requested amount to the actual requested amount $a$ and compute the likelihood function as

$$
\mathcal{L}(\theta)=\frac{1}{\sqrt{2 \pi v^{2}}} \exp \left(\frac{-(a-m)^{2}}{2 v^{2}}\right)
$$

Taking the natural logarithm of the likelihood, we obtain the log likelihood function as

$$
\ln (\mathcal{L}(\theta))=-\frac{1}{2} \ln \left(2 \pi v^{2}\right)-\frac{(a-m)^{2}}{2 v^{2}}
$$


To obtain the optimal value of the parameters $\mathcal{K}=\left\{\mu_{i}, \sigma_{i}, \mathrm{~S}, \mathrm{~T}\right\}$, we minimize the negative of the log-likelihood

$$
\min _{\mu_{i}, \sigma_{i}, \mathrm{~S}, \mathrm{~T}} \frac{1}{2} \ln \left(2 \pi v^{2}\right)+\frac{(a-m)^{2}}{2 v^{2}}
$$

We compute the parameters $\mathcal{K}=\left\{\mu_{i}, \sigma_{i}, \mathrm{~S}, \mathrm{~T}\right\}$ by coordinate gradient descent, keeping one parameter constant and obtaining the gradient of the other. The update equations for the parameters are given below

$$
\begin{aligned}
& \mu_{i} \leftarrow \mu_{i}+\eta \frac{a-m}{v}\left(\mathrm{qS}^{\mathrm{T}} \mathrm{TSc}^{\mathrm{T}} t_{i}\right) \\
& \sigma_{i} \leftarrow \sigma_{i}-\eta \frac{\sigma_{i}}{v^{2}}\left(v\left(1+\frac{a-m}{v}\right)-\left(\frac{a-m}{v}\right)^{2}\right) \\
& \mathrm{S} \leftarrow \mathrm{S}+\eta \frac{a-m}{v}\left(\mathrm{~T}+\mathrm{T}^{T}\right)\left(\mathrm{Sq}^{T} \sum_{i \in C} \mu_{i} t_{i} \mathbf{k}\right) \\
& \mathrm{T} \leftarrow \mathrm{T}+\eta \frac{a-m}{v}\left(\mathrm{Sq}^{T} \sum_{i \in C} \mu_{i} t_{i} \mathbf{u S}^{T}\right),
\end{aligned}
$$

We repeat this procedure for all the past requests and obtain optimal values of the parameter set $\mathcal{K}$ for the corresponding contributors.

For each new request in the test set, we obtain the candidate set $C$ corresponding to the request. We then compute the probable amount contributed by each contributor in the candidate set drawing from $\mathcal{N}\left(m_{i}, v_{i}\right)$ with mean $m_{i}$ and variance $v_{i}$ as

$$
m_{i}=\ln \left(s_{0}\right)+\left(\mu_{i} \mathbf{n}_{i}-\frac{\sigma_{i}^{2}}{2}\right) t_{i}, v_{i}=\sigma_{i}^{2} t .
$$

Here $s_{0}$ is the initial value of the contributor $i$ is willing to contribute to the platform and $t$ is the time elapsed since he first joined on the platform. The set $\mathcal{K}=\left\{\mu_{i}, \sigma_{i}, \mathrm{~S}, \mathrm{~T}\right\}$ are the optimal values of the parameters representing the financial information of the contributors computed from the past requests. We then sort the contributors in the candidate set in decreasing order of the probable contribution to the request and return to the requester as a ranked list. The procedure is summarized in Algorithm 1.

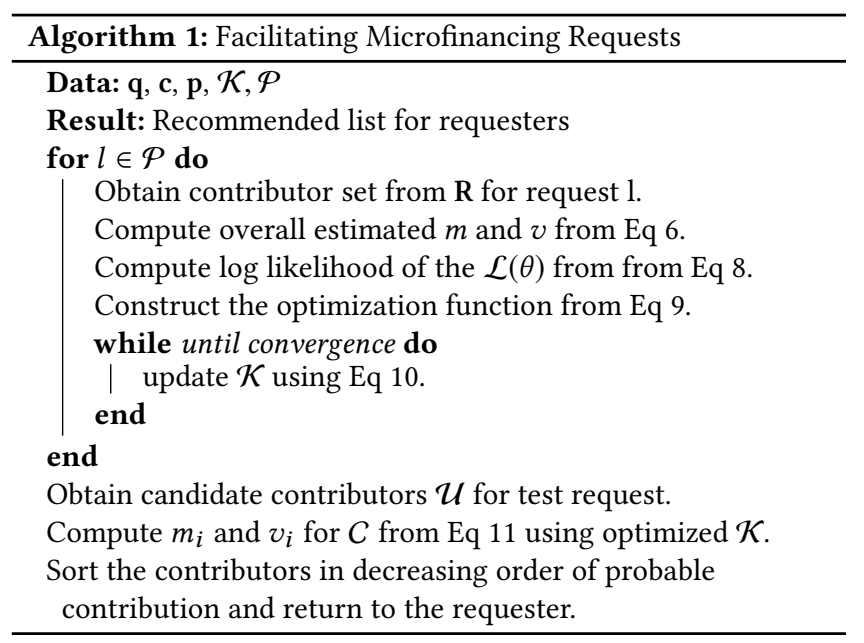

The inputs to the algorithm are the word-vector of the given request $\mathbf{q}$, the word-vectors of the interests of the contributor $\mathbf{c}$ and the past requests he has contributed towards $\mathbf{p}$, the parameter set
$\mathcal{K}$, and the training set of requests $\mathcal{P}$. The expected output of the algorithm is the ordered set of contributors recommended for the given request. For each request in the training set $\mathcal{P}$, we first obtain the list of contributors from the past request matrix $\mathbf{R}$. We estimate the requested amount by adding all the means and variances of the individual contributions of the list of contributors as shown in Eq 6. We compute the log-likelihood using Eq 8. We then maximize the $\log$ likelihood and update the value of the parameters in $\mathcal{K}$ until convergence using Eq 10. We repeat this procedure for all the past requests to obtain the optimal values of the parameter set $\mathcal{K}$.

We next use the parameter set to identify contributors to the given request as follows. We obtain the candidate contributor set $C$ for the given request. For each contributor $u_{i}$ in the candidate contributor set $C$, we estimate their probable contribution amount using Eq 11 from the optimal values of the parameter set $\mathcal{K}$. We sort the contributors in the candidate set according to the probable contribution of each contributor to the given request in descending order to return to the as a sorted list.

$$
\mathbf{e}_{\mathbf{t}}(q)=\sum_{i=1}^{o} \operatorname{sim}\left(\mathrm{qS}^{T} \mathbf{T}, \mathbf{S P}_{i}^{T}\right) \mathbf{r t}_{i},
$$

Until now, we described our framework to identify contributors for a given microfinancing request. We next compute the time complexity of the framework to demonstrate its scalability in large datasets usually prevalent in online microfinancing platforms.

\subsection{Time Complexity Analysis}

We now present the time-complexity to demonstrate the scalability of the framework for large datasets prevalent in online microfinancing platforms. The majority of the time complexity comes from the update equations in Eq 10. The complexity of $f_{i}=\mathrm{qS}^{\mathrm{T}} \mathrm{TSk}^{\mathrm{T}}$ is $O(W n)$. The complexity of $\left(\mathrm{T}+\mathrm{T}^{T}\right)\left(\mathrm{Sq}^{T} \sum_{i \in C} \mu_{i} t_{i} \mathbf{c}\right)$ is $O\left(W^{2} n\right)$. The complexity of $\mathrm{Sq}^{T}\left(\sum_{i \in C} \mu_{i} t_{i} \mathrm{cS}\right)$ is $O(W n)$. Therefore the overall complexity of the proposed algorithm is low owing to the sparsity of the word vectors and the low magnitude of latent dimension $n$, indicating the scalability of the framework to a large dataset.

In this section, we proposed a set of measures to correlate the requests and contributors with contributor interests and past response. We utilize the proposed measures to design financial models from the past implicit contributions to previous requests. We then utilize the financial models to identify contributors for new requests in online microfinancing platforms. In the next section, we present the collected data and experiments designed to evaluate our framework.

\section{EXPERIMENTAL EVALUATION}

In this section, we describe the dataset and use the dataset to evaluate our framework. We use the dataset to answer the following questions: How effective is the framework in identifying prospective contributors for requests in online microfinance platforms? How does the framework perform for requests with different amounts and number of contributors? How does the framework perform with varying proportions of training data size? We first present the dataset with some relevant analysis to understand some of its salient aspects. 


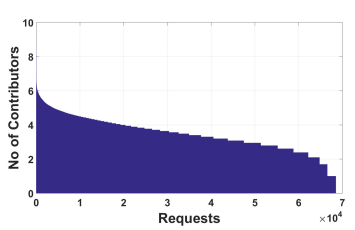

(a) No of Contributions per Request

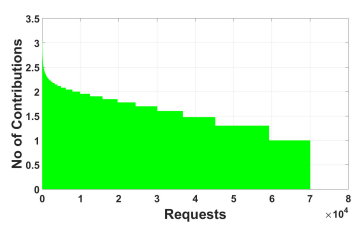

(e) No of Contributions per Requests

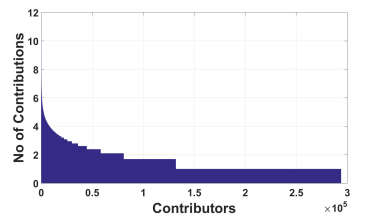

(b) No of Contributions

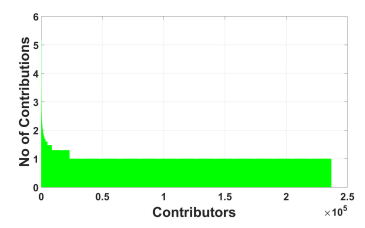

(f) No of Contributions

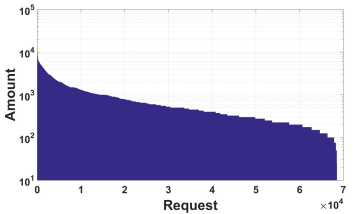

(c) Amount Requested

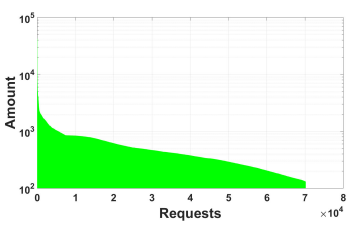

(g) Amount Asked per Request

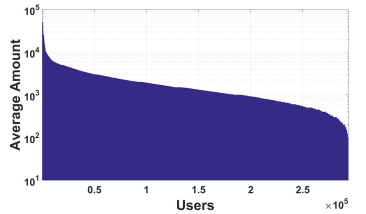

(d) Average Contributions of user

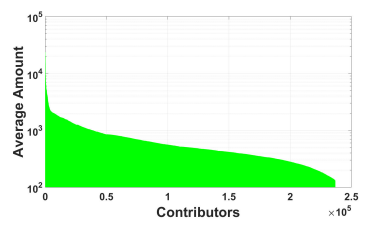

(h) Average Contributions of user

Figure 3: (a)-(d) Statistics of Kiva dataset (e)-(g) Statistics of DonorChoose dataset.

\begin{tabular}{lrr}
\hline Parameter & Kiva & DonorChoose \\
\hline \# Requests & 68,500 & 70,140 \\
\# Contributors & 293,736 & 232,562 \\
\# Contributions & $1,300,616$ & 370,053 \\
Median Contributions per request & 12 & 4 \\
Total amount requested & $\$ 54,148,425$ & $\$ 37,776,908$ \\
Median Amount requested & $\$ 475$ & $\$ 420$ \\
\hline
\end{tabular}

Table 1: Statistics of the datasets

\subsection{Data Analysis}

We collect the dataset from the microfinance platforms Kiva and DonorsChoose. Kiva provides a platform through which small entrepreneurs who are in need of funding can take the help of field partners like non-governmental organizations, while DonorsChoose focuses on asking donations to enable education infrastructure for teachers in need. The requests can be seen by potential contributors who can contribute part of the requested amount. Each request can be potentially fulfilled by a large number of individual contributors.

The statistics of the collected datasets is given in Table 1 . We describe the collected statistical data in the Kiva dataset. We collect 68,500 requests which have a total of 293,736 contributors. We select only successful requests to ensure optimal training of our algorithm. The total contributions across all requests are 1,300,616 with a median of 12 contributions per request. This indicates that requests in microfinancing platforms require a lot of contributors to get fulfilled. The total amount requested for is around $\$ 54$ million with a median amount of $\$ 475$ indicating the high activity of the site. Each request is accompanied by a brief description outlining the necessity for funding. Similarly, each contributor also describes himself outlining his purpose of being on the site and his primary interests. We also collected the request, and contributor descriptions and a total of 625,243 words are used in the requests and the contributors. Comparable statistics can be seen for all the parameters can be seen for the DonorsChoose datasets.
We next characterize various aspects of the dataset from Kiva and DonorsChoose datasets. The characterizations of the dataset regarding the traits of the contributors and requested amount are plotted in Figure 3. Figure 3(a) plots the number of contributors each request in the dataset has received. We take the natural logarithm of the number of contributions each request receives. We increment the values by 1 so that requests with a single contribution to emphasize the requests having a single contributor. We sort it in the descending order and plot it on the y-axis and plot the requests on the $x$-axis. From the figure, we can see that a few requests obtain a large number of contributors and a large number of requests receive a lesser number of contributors. We plot the number of contributions each contributor makes in Figure 3(b). Similar to Figure 3(a), we take the natural logarithm of the number of contributions each contributor makes, increment it by 1 . We plot this on the $y$-axis sorted in descending order and the corresponding contributors on the $\mathrm{x}$-axis. From the figure, we can see that a large percentage of contributors make a single contribution and a small number of contributors make a large number of contributions. We will evaluate the performance of the framework for requests with a varying number of contributors in Sec 4.4.

We next present characteristics of the dataset related to the amount requested. Figure 3(c) plots the amount asked by each request. The amount is sorted in descending order and plotted on the $\mathrm{y}$-axis, and the corresponding requests are plotted on the $\mathrm{x}$ axis. This follows a power law distributions where a few requests ask for large amounts while a large number of requests asking for lesser amounts of money. Overall, from the figure, we can see that a substantial amount of money is being requested across all requests on the platform. We next examine the requests contributed by each contributor and the amount asked by request. We then plot the average of the amounts asked for all the requests the contributor has contributed in Figure 3(d). From the figure, we can see that this too follows a power-law distribution with a few contributors contributing to requests with large amounts and also provides an indication of the varying contributions of different contributors. 
We will evaluate the performance of the framework for requests with a varying number of contributors in Sec 4.5.

Until now, we described the dataset and presented some analysis to understand some salient aspects of it. We now use the dataset and design experiments to evaluate our framework. We first describe the baseline we use to compare the performance of the framework.

\subsection{Experimental Settings}

In this work, we use the metrics Mean Average of Precision (MAP), Precision @K (Pre @K) and Mean Reciprocal Rank (MRR) to evaluate the performance of the different approaches. We compare the proposed framework against the following baselines:

- Random Selection: This method randomly select contributors from the dataset and the mean values of the evaluation metrics over 10 trails are taken. This is done to assess the difficulty of the problem.

- Sulprus Maximization[33]: The authors estimate the number of individual contributions using overall surplus maximization. We use the contributor interest to measure surplus. We use this baseline to evaluate the effectiveness of amount estimation for the proposed task.

- Topic Match: This baseline measures the matching between the latent representations of the contributor interests and requests as measured by Non-Negative Matrix Factorization [19]. We use the methods to assess the effectiveness of content matching to the proposed problem.

- Promotion [7]: The authors extract different features related to textual, contributor, requested amount, and time delay to identify useful projects for contributors. We use this work to evaluate the effectiveness of the features examined here for the proposed problem.

In the rest of the section, we describe experiments designed to evaluate the framework using the evaluation metrics. In Section 4.3 , we evaluate the performance of the framework in identifying contributors for requests on microfinance platforms. We divide the dataset into ten equal time epochs. We keep the first nine epochs for training and the last time epoch for testing. Next, we evaluate the robustness of the framework for varying contributor counts in Section 4.4, and varying requested amounts in Section 4.5.

\subsection{Identifying Contributors}

We now evaluate the performance of the framework in identifying contributors who can contribute effectively to funding requests in micro-financing platforms. We employ the procedure described in Algorithm 1 scoring the requests and the candidate contributors in the test set. For each request, we rank the candidate responders and employ the evaluation metrics to evaluate the performance of the framework in comparison to the baselines. The results of the experiments are presented in Table 2.

From the table, we can see that the performance of random ordering is low demonstrating the difficulty of the problem. The performance of Sulprus Maximization[33] improves upon the random performance showing the utility of estimating the individual contributions in identifying potential contributors. The improved performance of TopicMatch shows that the correlation of the contributors with the requests is a strong indicator for identifying contributors. The improved performance in Promotion[7] showcases the use of temporal and requested amount in identifying contributors to microfinance requests.

Our framework considerably outperforms existing baselines by a significant margin, thus demonstrating the utility of measuring the effect of past contribution amounts on user inclination for identifying contributors to online microfinance requests. This also demonstrates the effectiveness of the model in inferring implicit contribution amounts and modeling their effect on contributor inclination. We performed a paired t-test to compare the results with the baselines that showed that the improvement is significant with $p<0.001$.

In summary, we can say from Table 2 that our framework is effective in identifying contributors for requests posted in microfinancing platforms. Next, we will examine the effect on the performance of the framework due to variation in training data size and parameter values.

\subsection{Performance across Contributor Counts}

As observed during analysis of the data related to the contributor counts received by requests in Section 4.1, the number of contributors varies widely for different requests in the dataset. We now evaluate the performance of the framework for requests with a different number of contributors to evaluate its robustness. We plot the performance of the framework and the nearest baseline[7] in Fig 4(a) and Fig 4(b) using the MAP and Pre@10 metrics. We order the test requests in increasing number of contributors it obtained and plotted it on the $\mathrm{x}$-axis. For each contribution count, we compute the performance of the framework for requests having the same or lesser number of contributors and plot it on the y-axis. We present the results from the dataset from Kiva for the subsequent experiments to maintain better focus on the underlying concepts. We make the following observations from the two figures.

From Fig 4(a) and Fig 4(b), we notice that the framework outperforms the baselines for requests with a wide range of contributor counts. This demonstrates the robustness of the framework across requests with different counts of contributors. The framework performs relatively well with requests with a small number of contributors in both the metrics. The performance of the framework in MAP is lesser for requests with a higher number of contributors for both our framework and the baseline, showcasing the difficulty of the task when contributor count increases. From Section 4.1, we saw that the most requests have a small number of contributors, showing the effectiveness of the framework for identifying contributors for a wide range of requests.

In summary, the framework performs well for requests having a wide range of contributor counts and is robust to their variation, demonstrating its effectiveness for identifying contributors for different kinds of requests in microfinancing platforms. Next, we will examine the effect of the variation of the requested amount on the performance of the framework.

\subsection{Performance across Requested Amounts}

As observed during analysis of the data related to the requested amount asked by requests in Section 4.1, the requested amount 


\begin{tabular}{lcccccc}
\hline & \multicolumn{3}{c}{ Kiva } & \multicolumn{3}{c}{ DonorChoose } \\
Method & MAP & P@10 & MRR & MAP & P@ 10 & MRR \\
\hline Random & 0.1253 & 0.0921 & 0.2426 & 0.1892 & 0.0908 & 0.2499 \\
Sulprus Maximization [33] & 0.1377 & 0.1602 & 0.3289 & 0.1917 & 0.0948 & 0.2724 \\
TopicMatch & 0.2129 & 0.2064 & 0.4411 & 0.4419 & 0.1243 & 0.5943 \\
Promotion [7] & 0.2370 & 0.2612 & 0.4332 & 0.6670 & 0.2924 & 0.7384 \\
Our Method & $\mathbf{0 . 3 5 0 1}$ & $\mathbf{0 . 4 1 3 3}$ & $\mathbf{0 . 7 4 8 9}$ & $\mathbf{0 . 7 8 1 8}$ & $\mathbf{0 . 3 3 3 4}$ & $\mathbf{0 . 8 7 7 5}$ \\
\hline
\end{tabular}

Table 2: Performance evaluation of the framework. Our method outperforms baselines by a significant margin.

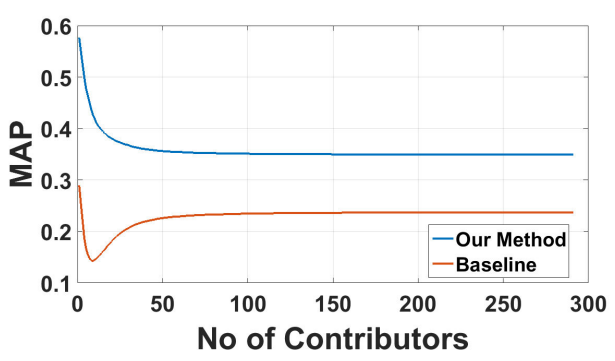

(a) MAP for Varying Amount

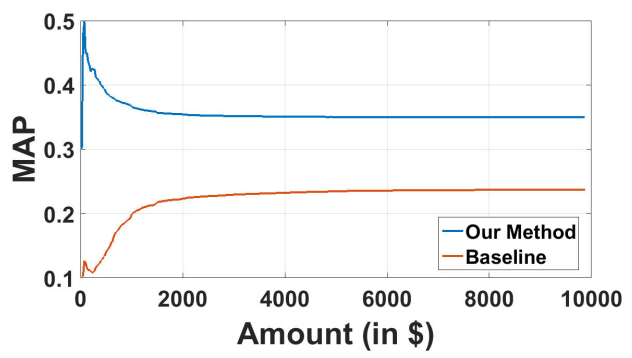

(c) MAP for Varying Contributor Count

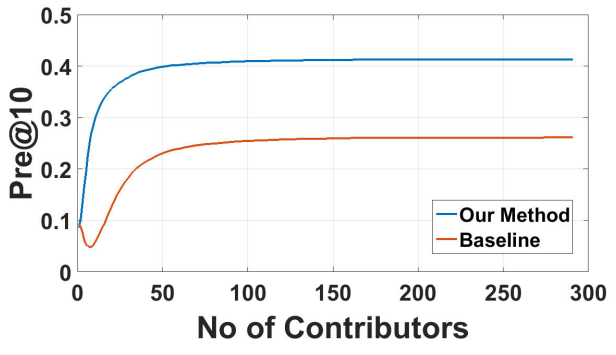

(b) Pre@10 for Varying Amount

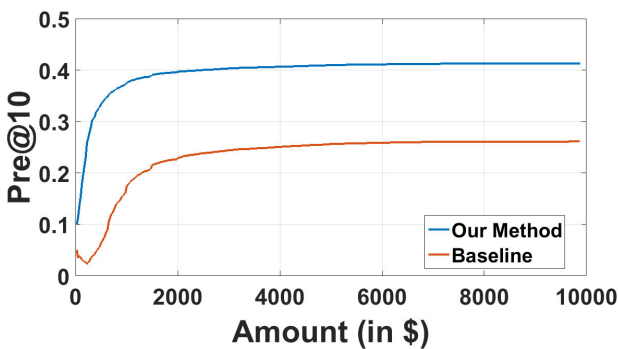

(d) Pre@10 for Varying Contributor Count

Figure 4: Performance of the framework at varying proportions of contributor count and requested amounts. The framework performs well for requests with different contributor counts and requested amounts.

varies widely across different kinds of requests. We now evaluate the performance of the framework across requests asking for various amounts. We then plot the performance of the framework and the nearest baseline[7] in Fig 4(c) and Fig 4(d) using the MAP and Pre@10 metrics. We order the test requests in increasing amount of requested contributions and plot it on the $\mathrm{x}$-axis. For each requested amount on the x-axis, we compute the performance of the framework for requests asking for the same or a lesser amount and plot it on the y-axis.

From Fig 4(c) and Fig 4(d), we notice that the framework outperforms the baselines for requests asking for a broad range of amounts. This demonstrates the robustness of the framework for identifying contributors for a wide variety of requests. The framework performs relatively well with requests requesting a small number of amounts for both the metrics. From Section 4.1, we saw that the most requests request small amounts, showing the effectiveness of the framework for identifying contributors for a wide class of requests in microfinancing problems.
In summary, the framework performs well for requests having a wide range of requested amounts and is robust to their variation, demonstrating its effectiveness for identifying contributors for different kinds of requests in microfinancing platforms. Next, we will examine the effect of the variation of the training data size on the performance of the framework.

\subsection{Effect of Variation in Training Data Size}

We now examine the change in the performance of the framework with varying proportions of training data. This enables us to examine the performance of the framework when less amount of training information is available and also assess the robustness of the framework with varying training data size. We divide the dataset into ten equal time periods. We keep the first nine epochs for training and the last time epoch for testing. We further train the framework with different proportions of the training data and evaluate the performance using the test dataset. The different proportions of the 


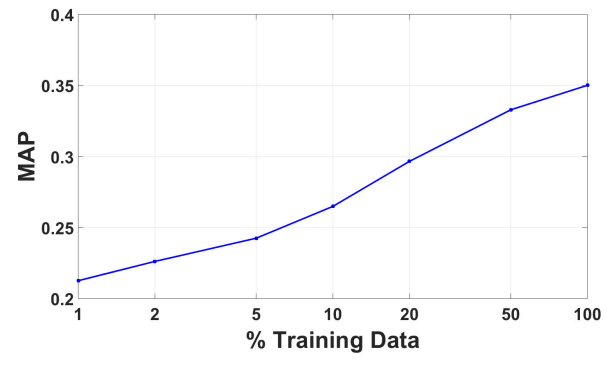

(a) MAP for Varying Training data proportion

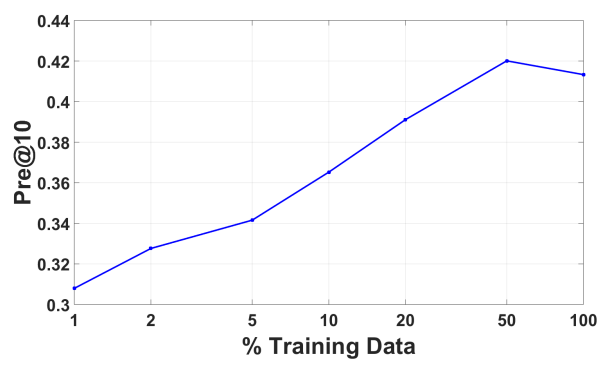

(b) Pre@10 for Varying Training data proportion

Figure 5: Performance of the framework at varying proportions of training data. The framework performs well at low proportions of training data.

training dataset we use are $\{1 \%, 2 \%, 5 \%, 10 \%, 20 \%, 50 \%, 100 \%\}$. We plot the results of the experiment in Fig 5(a) and 5(b) and make the following observations.

From the figure, we can say that the performance of the framework increases with increasing proportion of training data. The framework shows a good performance when $30 \%$ of the data is used for training, outperforming the nearest supervised baseline [21], demonstrating that it performs well for fairly low training data sizes. We observe a small dip in performance for higher proportions of training data, and this may be due to insufficient testing data. The performance increases with increasing training data, showing the ability of the framework to utilize the training data points effectively to identify contributors to requests posted in online microfinancing platforms.

In summary, the figure demonstrates that the framework performs well in the presence of small amount of training data and that the framework is effective in learning when more training data is available. This also demonstrates the effectiveness of the proposed framework in exploiting available financial information for identifying such contributors.

In this section, we evaluated the framework and compared it with existing baselines. We then examined the effect of variation of contributor count and requested amounts on its performance. We finally evaluated its performance in the presence of varying training data sizes. We next place our framework in the context of existing literature.

\section{RELATED WORK}

We present related literature in four categories: the study of resource seeking in social media, the recommendation in online microfinancing platforms, online microfinancing platforms, and Brownian motion models in online platforms.

Resource seeking in social media has received considerable attentions recently [11, 20,32]. An analytical study of the primary motivations for information seeking and responding in Twitter is presented in [22, 24]. A study of requests and responses received in Facebook [11] presented information seeking as a tool for resource mobilization in social media. Systems have been proposed to identify responders for social media requests to match request content with profile information [14] and use crowdsourced technology
[16]. A method for recommending users who can answer requests in social media [21] models temporal, behavioral and content related factors to identify suitable users. An unsupervised framework to identify users who can provide relevant answers for a given social media request is proposed in [29]. These works help social media users to find responders who can provide the information they need and do not address financial requests.

Identifying responders for online microfinancing requests have been addressed in recent literature $[8,33]$. A feature based approach based on contributor interest, profile and temporal information was designed to identify appropriate projects to communities [7]. These works provide an insight to design recommender systems for microfinance platforms but do not try to estimate and leverage past contribution amounts. Estimation of individual contribution amounts has been addressed in [33] by maximizing the overall surplus for the investor. These microfinance platforms do not provide interests to the contributors, and moreover, the effect of implicit contribution amounts on user inclination is not taken into consideration. We evaluate our framework using these models proposed in the literature as baselines and the experiments demonstrate that our framework significantly outperforms them.

Increasing attention has been devoted to characterizing user behavior in crowdfunding platforms [5, 6, 13]. The authors in [12] tracks the early rise of online microfinancing and stated that the access to a large number of potential donors could unleash the entrepreneurship of the poor. The motivations of contributors to contribute money is studied in [15] and the desire to help others and be a part of a cause is stated to be a common motivator for contributors. The donor retention in online crowdfunding platforms has been studied in [2] and positive interaction and acknowledgment of their support are found to be predictive of returning donors. Algorithmic systems for crowdfunding domains proposed in the literature include connecting contributors to appropriate teams [6], using communities to identify projects for contributors [7], and identifying appropriate projects to groups of contributors [27].

The study of Brownian motion on online platforms has been investigated in [34-36]. The theoretical development for adapting Brownian motion in the network environment has been considered in [36], with applications in community discovery. Brownian motion has been used to model information propagation processes 
in the presence of external influence in social networks [17, 28] and the authors model the diffusion process across the propagation network to predict protest participation. Brownian motion models have been used to model financial time series data, including model movements of stock price due to its ability to model sharp changes [31] and to model variation in commodity prices due to change in contributor preferences over time [9].

\section{CONCLUSIONS AND FUTURE WORK}

Existing microfinancing platforms deal with a large frequency of funding requests, preventing people seeking financial assistance from getting appropriate assistance from potential contributors. Fulfilling financial requests has a cost on contributors which can affect his inclination to contribute. We draw from financial modeling literature to propose a novel framework to identify potential contributors in microfinancing platforms by modeling the effect of implicit individual contribution amounts on his inclination to contribute. We evaluate the framework on two microfinancing platforms to demonstrate its effectiveness in identifying contributors. The robustness of the framework has been shown with varying proportions of information related to contribution counts and requested amounts. We finally evaluate the ability of our framework to identify contributors in varying proportions of training data and demonstrate its effectiveness in the presence of less information.

For future work, techniques such as word-to-vec which are effective for low-dimensional representation, can be integrated into the algorithm to better address novel requests containing words not seen during training. Incorporating features like teams, contributor and request profile information to estimate amounts can further improve the performance of the framework. Estimating contribution amounts considering network effects will provide insight into how contributors are influenced by the donation behavior of their neighbors. Evaluating the privacy issues in leveraging past contribution amounts can shed lights on the characteristics of user vulnerability on microfinancing platforms. Modeling the effect of competition among requesters for identifying contributors will bring new insights on how people compete in a resource constrained environment. Predicting requesters who are prone not to repay the loan will help in increasing the satisfaction of the contributors and increase their faith in the microfinancing platform.

\section{ACKNOWLEDGMENTS}

This material is based upon work supported by, or in part by, Office of Naval Research (ONR) under grant numbers N000141410095 and N00014-16-1-2257.

\section{REFERENCES}

[1] Wei Ai, Roy Chen, Yan Chen, Qiaozhu Mei, and Webb Phillips. 2016. Recommending teams promotes prosocial lending in online microfinance. Proceedings of the National Academy of Sciences 113, 52 (2016), 14944-14948.

[2] Tim Althoff and Jure Leskovec. 2015. Donor retention in online crowdfunding communities: A case study of donorschoose. org. In WWW. ACM.

[3] Jisun An, Daniele Quercia, and Jon Crowcroft. 2014. Recommending investors for crowdfunding projects. In $W W W$. ACM.

[4] Paul N Bloom. 2009. Overcoming consumption constraints through social entrepreneurship. Fournal of Public Policy \& Marketing 28, 1 (2009), 128-134.

[5] Tillman Bruett et al. 2007. Cows, Kiva, and Prosper. Com: how disintermediation and the internet are changing microfinance. Community Development Investment Review 2 (2007), 44-50.
[6] Roy Chen, Yan Chen, Yang Liu, and Qiaozhu Mei. 2015. Does team competition increase pro-social lending? Evidence from online microfinance. Games and Economic Behavior (2015).

[7] Jaegul Choo, Changhyun Lee, Daniel Lee, Hongyuan Zha, and Haesun Park. 2014. Understanding and promoting micro-finance activities in kiva. org. In WSDM. ACM.

[8] Jaegul Choo, Daniel Lee, Bistra Dilkina, Hongyuan Zha, and Haesun Park. 2014. To gather together for a better world: understanding and leveraging communities in micro-lending recommendation. In $W W W$. ACM.

[9] Shijie Deng. 2000. Stochastic models of energy commodity prices and their applications: Mean-reversion with jumps and spikes. Berkeley: University of California Energy Institute.

[10] Merel Van Diepen, Bas Donkers, and Philip Hans Franses. 2009. Dynamic and competitive effects of direct mailings: A charitable giving application. fournal of Marketing Research 46, 1 (2009), 120-133.

[11] Nicole B Ellison, Rebecca Gray, Jessica Vitak, Cliff Lampe, and Andrew T Fiore. 2013. Calling All Facebook Friends: Exploring Requests for Help on Facebook.. In International AAAI Conference on Web and Social Media.

[12] Matt Flannery. 2007. Kiva and the birth of person-to-person microfinance. Innovations 2, 1-2 (2007), 31-56.

[13] Jeff Galak, Deborah Small, and Andrew T Stephen. 2011. Microfinance decision making: A field study of prosocial lending. Journal of Marketing Research 48, SPL (2011), S130-S137.

[14] Brent Hecht, Jaime Teevan, Meredith Morris, and Dan Liebling. 2012. SearchBuddies: Bringing Search Engines into the Conversation. In ICWSM.

[15] Julie S Hui, Michael D Greenberg, and Elizabeth M Gerber. 2014. Understanding the role of community in crowdfunding work. In CSCW. ACM.

[16] Jin-Woo Jeong, Meredith Ringel Morris, Jaime Teevan, and Dan Liebling. 2013. A Crowd-Powered Socially Embedded Search Engine. In ICWSM.

[17] Fang Jin, Rupinder Paul Khandpur, Nathan Self, Edward Dougherty, Sheng Guo, Feng Chen, B Aditya Prakash, and Naren Ramakrishnan. 2014. Modeling mass protest adoption in social network communities using geometric brownian motion. In KDD. ACM.

[18] Ioannis Karatzas and Steven Shreve. 2012. Brownian motion and stochastic calculus. Vol. 113. Springer Science \& Business Media.

[19] Daniel D Lee and H Sebastian Seung. 2001. Algorithms for non-negative matrix factorization. In NIPS.

[20] Uichin Lee, Hyanghong Kang, Eunhee Yi, Mun Yi, and Jussi Kantola. 2012. Understanding mobile q\&a usage: An exploratory study. In CHI. ACM.

[21] Jalal Mahmud, Michelle Zhou, Nimrod Megiddo, Jeffrey Nichols, and Clemens Drews. 2014. Optimizing the selection of strangers to answer questions in social media. arXiv preprint arXiv:1404.2013 (2014).

[22] Meredith Ringel Morris, Jaime Teevan, and Katrina Panovich. 2010. What do people ask their social networks, and why?: a survey study of status message q\&a behavior. In CHI. ACM.

[23] Bernt Oksendal. 2003. Stochastic differential equations. In Stochastic differential equations. Springer, 65-84.

[24] Sharoda A Paul, Lichan Hong, and Ed H Chi. 2011. Is Twitter a Good Place for Asking Questions? A Characterization Study. In ICWSM.

[25] Craig Pirrong. 2011. Commodity price dynamics: A structural approach. Cambridge University Press.

[26] Ioana Popescu and Yaozhong Wu. 2007. Dynamic pricing strategies with reference effects. Operations Research 55, 3 (2007), 413-429.

[27] Vineeth Rakesh, Wang-Chien Lee, and Chandan K Reddy. 2016. Probabilistic Group Recommendation Model for Crowdfunding Domains. In WSDM. ACM.

[28] Suhas Ranganath, Fred Morstatter, Xia Hu, Jiliang Tang, Suhang Wang, and Huan Liu. 2016. Predicting Online Protest Participation of Social Media Users. In AAAI.

[29] Suhas Ranganath, Jiliang Tang, Xia Hu, Hari Sundaram, and Huan Liu. 2015. Leveraging Social Foci for Information Seeking in Social Media. In AAAI.

[30] Jacob Solomon, Wenjuan Ma, and Rick Wash. 2016. Highly Successful Projects Inhibit Coordination on Crowdfunding Sites. In CHI. ACM.

[31] Peter Tankov. 2003. Financial modelling with jump processes. Vol. 2. CRC press.

[32] Jiang Yang, Meredith Ringel Morris, Jaime Teevan, Lada A Adamic, and Mark S Ackerman. 2011. Culture Matters: A Survey Study of Social Q\&A Behavior. ICWSM (2011).

[33] Yongfeng Zhang, Qi Zhao, Yi Zhang, Daniel Friedman, Min Zhang, Yiqun Liu, and Shaoping Ma. 2016. Economic recommendation with surplus maximization. In $W W W$.

[34] Haijun Zhou. 2003. Distance, dissimilarity index, and network community structure. Physical review 67, 6 (2003), 061901.

[35] Haijun Zhou. 2003. Network landscape from a Brownian particle's perspective. Physical Review E 67, 4 (2003), 041908.

[36] Haijun Zhou and Reinhard Lipowsky. 2004. Network brownian motion: A new method to measure vertex-vertex proximity and to identify communities and subcommunities. In Computational Science-ICCS 2004. Springer, 1062-1069. 